\title{
Mechanism of osteogenic and adipogenic differentiation of tendon stem cells induced by sirtuin 1
}

\author{
JUNPENG LIU ${ }^{1}$, WEIFENG HAN ${ }^{2}$, LEI CHEN $^{3}$ and KANGLAI TANG ${ }^{1}$ \\ ${ }^{1}$ Department of Orthopaedic Surgery, Southwest Hospital, Third Military Medical University, Chongqing 400038; \\ ${ }^{2}$ Department of Orthopaedic Surgery, Beijing Tian Tan Hospital, Capital Medical University, Hangzhou, Zhejiang 310003; \\ ${ }^{3}$ Department of Orthopaedic Surgery, Wuhan General Hospital of Guangzhou Military Area in Wuhan, \\ Wuhan, Hubei 430070, P.R. China
}

Received December 16, 2014; Accepted December 29, 2016

DOI: $10.3892 / \mathrm{mmr} .2016 .5417$

\begin{abstract}
The aim of the present study was to assess the expression of sirtuin (Sirt) 1 in tendon stem cells (TSCs) and to elucidate its association with osteogenic and adipogenic differentiation of TSCs. Reverse-transcription quantitative polymerase chain reaction (RT-qPCR) and western blot analyses were performed to detect Sirtl mRNA and protein levels in TSCs, respectively. TSCs were positive for Sirt1 expression, which was elevated by Sirt1 activator SRT1720 in a time- and concentration- dependent manner, and decreased by Sirt1 inhibitor EX527. TSCs were treated with SRT1720 and EX527 for various time periods and resulting changes in osteogenic and adipogenic protein markers were analyzed using alizarin red and oil red $\mathrm{O}$ staining. According to RT-qPCR and western blot analyses, the associated factors $\beta$-catenin, Runt-related transcription factor 2 (Runx2) and bone morphogenetic protein 2 were elevated following increases of Sirt1 levels, while CCAAT/enhancer binding protein (CEBP) $\alpha$ and peroxisome proliferator-activated receptor (PPAR) $\gamma$ were decreased. These results suggested that osteogenic differentiation capacity was enhanced, while adipogenic differentiation capacity declined. Further mechanistic study revealed that phosphoinositide-3 kinase (PI3K) and AKT were decreased following activation of Sirt1. In conclusion, the present study suggested that Sirt1 promotes the osteogenic differentiation of TSCs through upregulating $\beta$-catenin and Runx 2 and inhibits the adipogenic differentiation of TSCs through the PI3K/AKT pathway with downregulation of CEBP $\alpha$ and PPAR $\gamma$.
\end{abstract}

Correspondence to: Professor Kanglai Tang, Department of Orthopaedic Surgery, Southwest Hospital, Third Military Medical University, 30 Gao Tanyan Street, Shapingba, Chongqing 400038, P.R. China

E-mail: tangkanglai@126.com

Key words: tendon stem cells, sirtuin 1, osteogenic differentiation, adipogenic differentiation, Runt-related transcription factor 2, peroxisome proliferator-activated receptor $\gamma$

\section{Introduction}

Tendinopathy is a strain-induced chronic disease whose causes remain to be elucidated. The frequency of cases of tendinopathy is increasing, and it currently accounts for $\sim 50 \%$ of all sports injuries (1). As conservative treatments are limited, they are unsuccessful in $24-45.5 \%$ of patients, leading to refractory tendinopathy (2). Therefore, an enhanced understanding of the pathogenesis of tendinopathy at the cellular and molecular level is required to develop novel and more effective treatment strategies.

Tendon stem cells (TSCs) are a type of mesenchymal stem cells derived from the paratenon of the tendon. Zhang and Wang (3) indicated that TSCs have a higher potential for cell proliferation and collagen synthesis as compared with that of mature tendon cells. Under certain conditions, TSCs can be differentiated into mature tendon cells with an enhanced secretion of extracellular matrix to effectively repair tendon injury (3). However, the underlying mechanisms of TSC differentiation have remained elusive. TSCs are pluripotent and thus capable of differentiating into adipocytes, chondrocytes and osteoblasts (4-8). Their multi-directional differentiation potential has an important role in the process of tendon repair. Insight into the regulation of TSC differentiation may provide novel strategies for the treatment of tendinopathy, as these cells may be utilized for tendon injury repair.

Sirt1 is a nicotinamide adenine dinucleotide $\left(\mathrm{NAD}^{+}\right)$-dependent histone deacetylase, which has an important role in energy metabolism and cell differentiation (9). A study has shown that Sirtl can regulate osteogenic differentiation by self-deacetylation in mesenchymal stem cells (10). Resveratrol, an activator of Sirt1, can decrease the number of adipocytes and increase the expression of osteoblast markers (11). Inhibition of Sirt1 can increase the number of adipocytes and lipoblast markers and reduce the expression of osteoblast markers. Thus, the activation of Sirt1 can inhibit adipogenic differentiation and promote osteogenic differentiation (11). However, the expression status of Sirt1 in TSCs and the underlying mechanisms of its possible regulation of osteogenic and adipogenic differentiation in TSCs have remained elusive.

Using reverse-transcription quantitative polymerase chain reaction (RT-qPCR) and western blot analyses, the present 
study assessed the gene and protein expression of Sirtl in TSCs following treatment with a Sirt1 activator or inhibitor for various time periods. In addition, the present study assessed the resulting differentiation into osteoblasts and lipoblasts and elucidated the molecular mechanism of Sirt1-mediated regulation of osteogenic and adipogenic differentiation of TSCs. The present study provided insight into the molecular mechanisms of Sirt1-induced TSC differentiation, which may be harnessed for the development of novel and effective treatments for tendon injury.

\section{Materials and methods}

Isolation and culture of rat TSCs. Three eight-week-old Sprague-Dawley male rats weighing 250-300 g were purchased from the Shanghai Institute of Materia Medica, Chinese Academy of Science (Shanghai, China) and used in the present study. All experiments were approved by the Animal Research Ethics Committee of the Third Military Medical University (Chongqing, China). The isolation and culture of rat TSCs were performed as described previously (12-14). Culture medium with or without Sirt1 activator SRT1720 (0.04 or $0.02 \mu \mathrm{M}$; Sigma-Aldrich, St. Louis, MO, USA) or Sirt1 inhibitor EX527 (0.02 $\mu \mathrm{M}$; Santa Cruz Biotechnology, Inc., Dallas, TX, USA) was changed every three days throughout the experiments. Analyses were performed on days 1, 3, 7, 10 and 14 of incubation.

$R T$ - $q P C R$. RNA was extracted with TRIzol reagent (Invitrogen; Thermo Fisher Scientific Inc., Waltham, MA, USA; $1 \mathrm{ml}$ per $1 \times 10^{7}$ cells) and cDNA was synthesized using the RNA reverse transcription kit (cat no. DRR047A; Takara, Dalian, China) according to the manufacturer's instructions. The sequences of the Sirt1 gene primers were 5'-CCAGATCCTCAAGCC ATGT-3' for the forward primer and 5'-TTGGATTCCTGC AACCTG-3' for the reverse primer. The sequences of the Runx2 gene primers were 5'-TGATGACACTGCCACCTC TGACTT-3' for the forward primer and 5'-TGGATAGTGCAT TCGTGGGTTGGA-3' for the reverse primer. The sequences of the PPAR $\gamma$ gene primers were 5'-ATGACCACTCCCATT CCTTT-3' for the forward primer and 5'-TGATCGCACTTT GGTATTCTT-3' for the reverse primer. The sequences of the GAPDH primers were 5'-ATGGAAATCCCATCACCATCT T-3' for the forward primer and 5'-CGCCCCACTTGATTT TGG-3' for the reverse primer. The primers were obtained from Sangon Biotech Co. Ltd. (Shanghai, China). A total of $5 \mu 1$ cDNA from each sample was obtained and PCR was performed in $25 \mu \mathrm{l}$ reactions containing Platinum SYBR Green qPCR SuperMix-UDG and appropriate primers in the ABI StepOne Plus system from Applied Biosystems (Foster City, CA, USA). Cycling parameters were as follows: Denaturation, $95^{\circ} \mathrm{C}$ for 10 min followed by 45 cycles consisting of denaturation at $95^{\circ} \mathrm{C}$ for $20 \mathrm{sec}$, annealing at $60^{\circ} \mathrm{C}$ for $25 \mathrm{sec}$, extension at $72^{\circ} \mathrm{C}$ for $30 \mathrm{sec}$, and a controlled increase to $95^{\circ} \mathrm{C}$ with a heating rate of $0.1^{\circ} \mathrm{C} / \mathrm{sec}$. Target gene expression was normalized to that of GAPDH. Data were analyzed using the comparative threshold cycle $(\mathrm{Cq})$ method. The melting curves were analyzed after amplification. PCR reactions of each sample were performed in triplicate. Data were analyzed using the comparative $2^{-\Delta \Delta C q}$ method.
Protein extraction and western blot analysis. Cells were scraped and homogenized in lysis buffer (Beyotime Institute of Biotechnology, Haimen, China) containing a mixture of proteinase inhibitors (Thermo Fisher Scientific Inc.). Protein concentrations were measured using a bicinchoninic acid protein assay kit (Beyotime Institute of Biotechnology). Protein samples (40 $\mu \mathrm{g} /$ lane) were resolved by $10 \%$ SDS-PAGE and transferred onto polyvinylidene difluoride membranes. After blocking with $0.1 \%$ Tris-buffered saline containing Tween 20 (Beyotime Institute of Biotechnology) and 5\% non-fat milk for $1 \mathrm{~h}$ at $20^{\circ} \mathrm{C}$, membranes were incubated at $4^{\circ} \mathrm{C}$ for $1 \mathrm{~h}$ sequentially with primary and secondary antibodies. The following primary antibodies purchased from Abcam (Cambridge MA, USA) were used: Anti-Sirt1 (monoclonal mouse IgG; 1:1,000 dilution; cat. no. ab50517), anti- $\beta$-catenin (monoclonal mouse IgG; 1:800 dilution; cat. no. ab22656), anti-Runt-related transcription factor 2 (Runx2; monoclonal mouse IgG; 1:1,000 dilution; cat. no. ab115899), anti-CCAAT/enhancer binding protein (CEBP) $\alpha$ (monoclonal mouse IgG; 1:1,000 dilution; cat. no. ab128482) and anti-peroxisome proliferator-activated receptor (PPAR) $\gamma$ (monoclonal mouse IgG; 1:1200 dilution; cat. no. ab138004), anti-PI3K (monoclonal mouse; 1:1,000 dilution; cat. no. ab86714), anti-AKT (monoclonal rabbit IgG; 1:1,500 dilution; cat. no. ab32505), anti-BMP2 (monoclonal rabbit IgG; 1:1,000 dilution; cat. no. ab183729). Subsequently, the membranes were incubated at room temperature for $2 \mathrm{~h}$ with peroxidase-labeled anti-mouse $\operatorname{IgG}$ antibody $(1: 1,000$ dilution; cat. no. A0216; Beyotime Institute of Biotechnology) or anti-rabbit IgG antibody (1:1,000 dilution; cat. no. A0208; Beyotime Institute of Biotechnology). Proteins were visualized using an enhanced chemiluminescence (ECL) reagent (Amersham ECL Prime detection; GE Healthcare, Little Chalfont, UK). Experiments were performed in triplicate.

Alizarin red staining. Cells were fixed in $70 \%$ ethanol for $1 \mathrm{~h}$ and stained with $2 \%$ alizarin red solution (Beyotime Institute of Biotechnology; $\mathrm{pH} 4.1-4.5$ ) for $30 \mathrm{~min}$ at $20^{\circ} \mathrm{C}$. The stained cells were examined under an inverted microscope (ix71; Olympus Corporation, Tokyo, Japan). Photomicrographs were obtained using a charge-coupled device camera.

Oil Red $O$ staining. Adipocytes were identified by staining with Oil Red O (Sigma-Aldrich) as described previously (15). The area of the cells stained with Oil Red $\mathrm{O}$ was measured using ImagePro Plus 6.0 software (Media Cybernetics, Rockville, MD, USA).

Statistical analysis. SPSS version 17.0 (SPSS, Inc, Chicago, IL, USA) software was used for statistical analysis. Values are expressed as the mean + standard deviation. Multiple comparisons were made using one-way analysis of variance followed by Fisher's exact test. $\mathrm{P}<0.05$ was considered to indicate a statistically significant difference between values.

\section{Results}

Sirtl is expressed in TSCs and drives osteogenic differentiation. In order to investigate the association between Sirt1 and the osteogenic and adipogenic differentiation of TSCs, the present study first detected Sirt1 mRNA and protein 
A

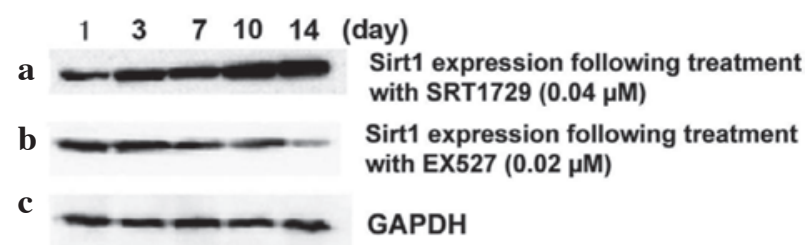

B

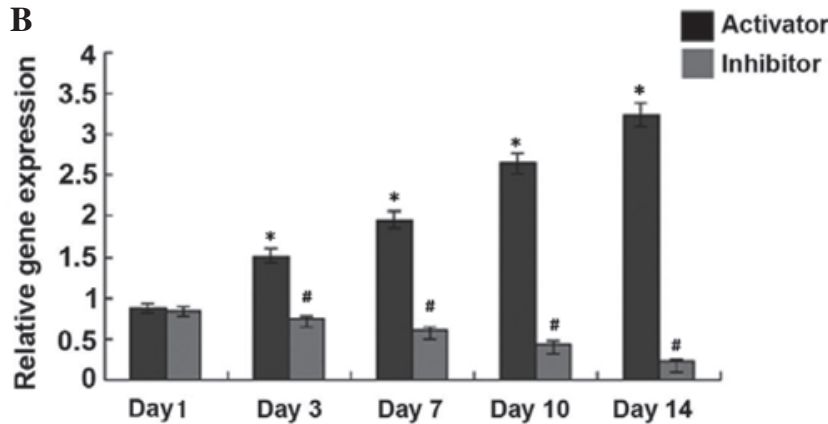

C

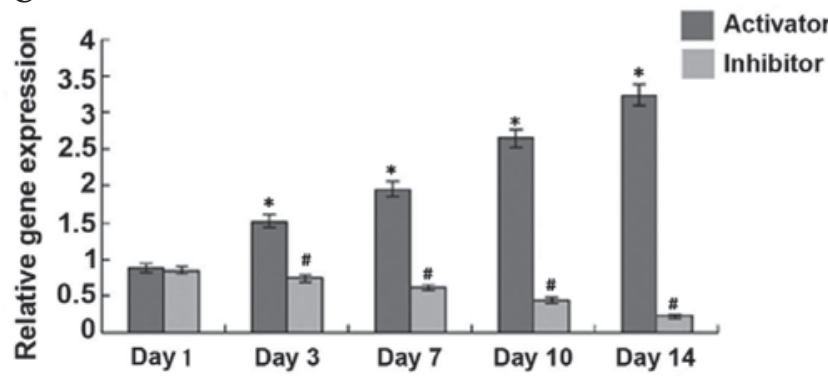

Figure 1. Expression of Sirt1 in TSCs. (Aa and Ab) Expression of Sirt1 in TSCs on days 3, 7, 10 and 14 following treatment with the Sirt1 activator SRT1720 $(0.4 \mu \mathrm{M})$ or inhibitor EX527 $(0.02 \mu \mathrm{M})$. (Ac) Expression of GAPDH. (B) Protein expression levels of Sirt1 were quantified by grey value analysis relative to GAPDH. (C) Reverse transcription quantitative polymerase chain reaction analysis of Sirt1 mRNA expression following treatment with SRT1720 $(0.4 \mu \mathrm{M})$ and EX527 $(0.02 \mu \mathrm{M})$, respectively. ${ }^{*} \mathrm{P}<0.05$, compared with the control group treated with SRT1729; ${ }^{\text {\# }} \mathrm{P}<0.05$, compared with the control group treated with EX527. Values are expressed as the mean \pm standard deviation. TSC, tendon stem cell; Sirt, sirtuin.

expression in TSCs. As shown in Fig. 1, TSCs expressed Sirt1, which was further enhanced by Sirt1 activator SRT1720 in a concentration- and time-dependent manner. Conversely, Sirt1 expression decreased in the presence of Sirt1 inhibitor EX527 in a time-dependent manner $(\mathrm{P}<0.05)$. Next, the present study evaluated the expression of adipogenic and osteogenic differentiation markers in TSCs on days 3, 7, 10 and 14 of treatment with Sirt1 activator and inhibitor. The expression of BMP2 and Runx2 appeared to be positively correlated with Sirt1 expression, which indicated that Sirt1 increases the capacity of TSCs for osteogenic differentiation (Fig. 2). Peak values of BMP2 and Runx 2 expression were observed on day 10, followed by a marked decrease in osteogenic differentiation capacity of TSCs during prolonged treatment with Sirtl activator. However, the adipogenic differentiation capacity of TSCs was gradually reduced $(\mathrm{P}<0.05)$.

Sirt1 enhances osteogenic and decreases adipogenic differentiation of tendon stem cells. Alizarin red staining was used to assess the osteogenic differentiation and oil red $\mathrm{O}$ staining was used to detect the adipogenic differentiation of tendon stem cells after treatment with SRT1720 $(0.4 \mu \mathrm{M})$ (Fig. 3). In
A
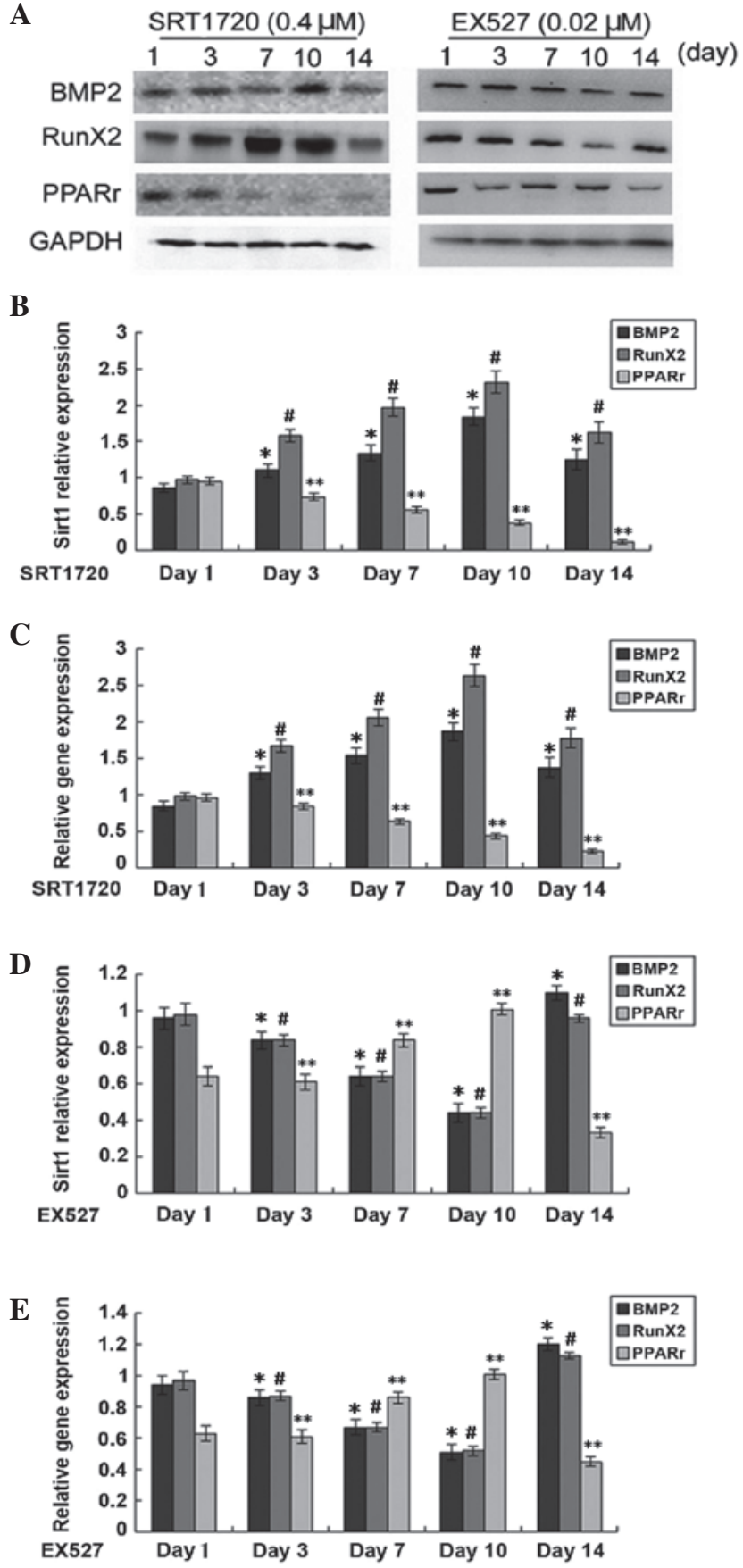

Figure 2. Expression of marker proteins of osteogenic and adipogenic differentiation on days 3, 7, 10 and 14 after treatment with sirtuin 1 activator SRT1720 $(0.4 \mu \mathrm{M})$ and inhibitor EX527 $(0.02 \mu \mathrm{M})$. (A) Representative western blots of BMP2, RunX2 and PPAR $\gamma$. (B) Protein levels following SRT1720 treatment were quantified by grey value analysis of A with normalization to GAPDH. (C) Reverse-transcription quantitative polymerase chain reaction analysis of mRNA levels of BMP2, RunX2 and PPAR $\gamma$ following SRT1720 treatment. (D) Protein levels following EX527 treatment were quantified by grey value analysis of A with normalization to GAPDH. (E) Reverse-transcription quantitative polymerase chain reaction analysis of mRNA levels of BMP2, RunX2 and PPAR $\gamma$ following EX527 treatment. Values are expressed as the mean \pm standard deviation. ${ }^{*} \mathrm{P}<0.05$, compared with the control group (BMP2); ${ }^{\text {"}} \mathrm{P}<0.05$, compared with the control group (RunX2); ${ }^{* *} \mathrm{P}<0.05$, compared with the control group (PPAR $\gamma$ ). BMP, bone morphogenetic protein; RunX2, Runt-related transcription factor 2; PPAR, peroxisome proliferator-activated receptor.

accordance with the effects of Sirt1 on the expression of differentiation markers (Fig. 2), staining showed that the capacity 


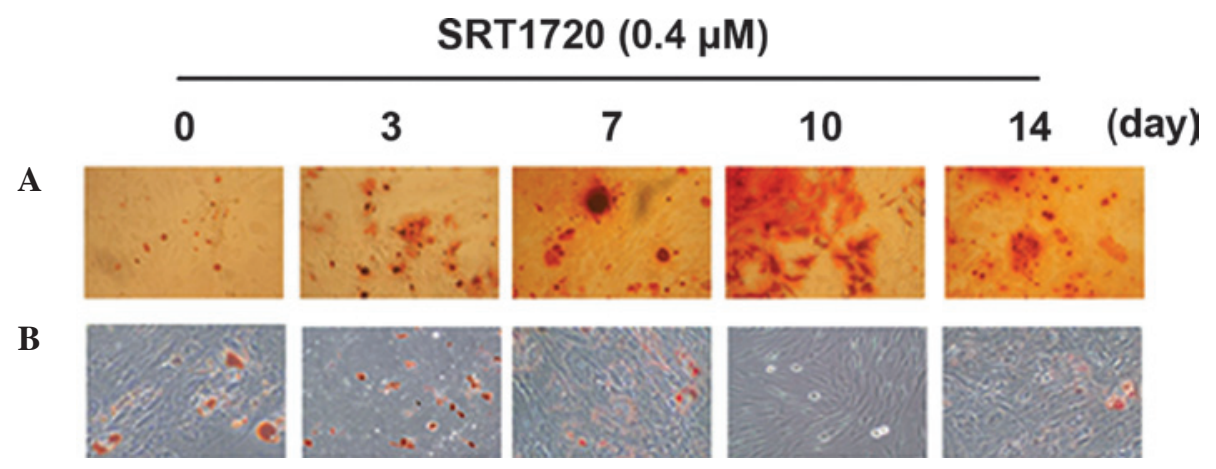

Figure 3. Osteogenic and adipogenic differentiation of tendon stem cells after treatment with sirtuin1 activator SRT1720 (0.4 $\mu \mathrm{M})$. (A) Alizarin red staining was used to observe osteogenic differentiation. (B) Oil red O staining was used to detect adipogenic differentiation of tendon stem cells. Original magnification, $\mathrm{x} 40$.
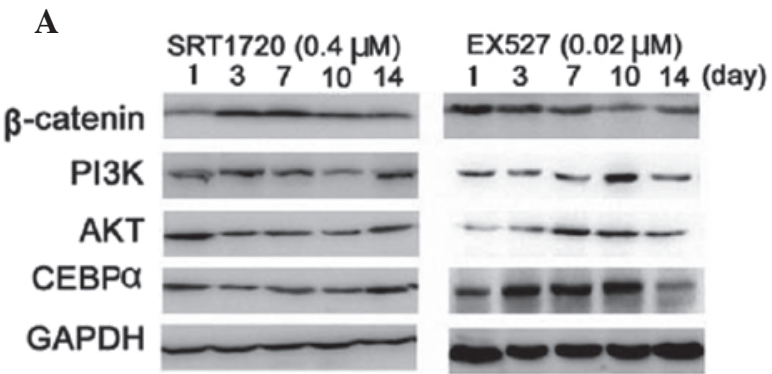

B

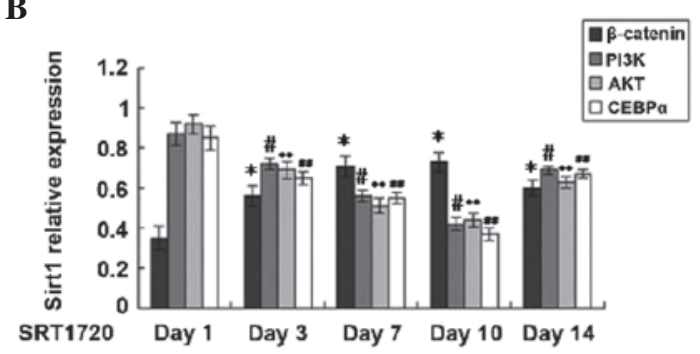

D

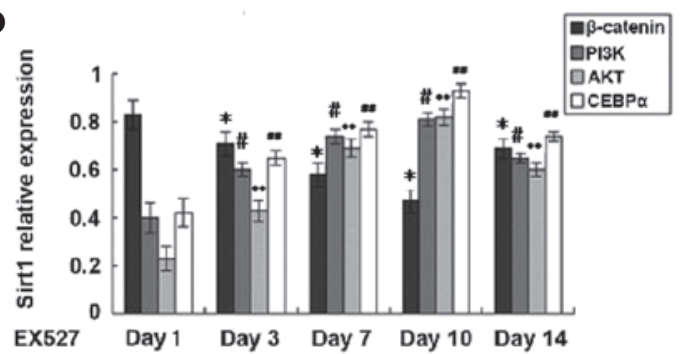

C

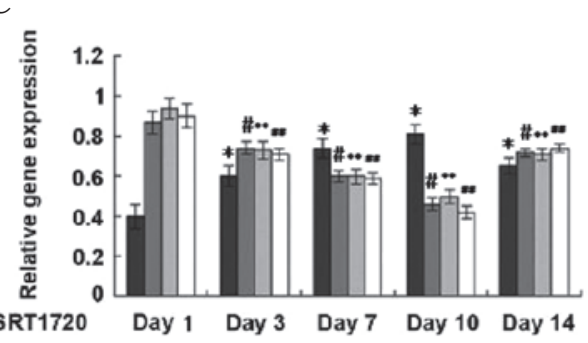

$\mathbf{E}$

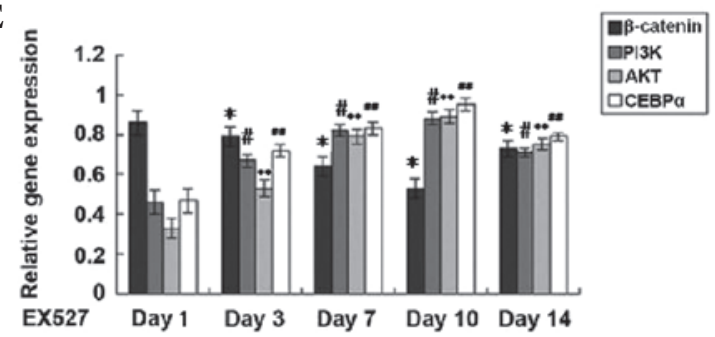

Figure 4. Expression of proteins and genes involved in signaling pathways on days 3, 7, 10 and 14 of treatment with sirtuin 1 activator SRT1720 (0.4 $\mu \mathrm{M})$ or inhibitor EX527 $(0.02 \mu \mathrm{M})$. (A) Representative western blots of $\beta$-catenin, PI3K, AKT and CEBP $\alpha$. (B) Protein levels following SRT1720 treatment were quantified by grey value analysis of A with normalization to GAPDH. (C) Reverse-transcription quantitative polymerase chain reaction analysis of mRNA levels of $\beta$-catenin, PI3K, AKT and CEBP $\alpha$ following SRT1720 treatment. (D) Protein levels following EX527 treatment were quantified by grey value analysis of A with normalization to GAPDH. (E) Reverse-transcription quantitative polymerase chain reaction analysis of mRNA levels of $\beta$-catenin, PI3K, AKT and CEBP $\alpha$ following EX527 treatment. Values are expressed as the mean \pm standard deviation. ${ }^{*} \mathrm{P}<0.05$, compared with the control group ( $\beta$-catenin); ${ }^{\#} \mathrm{P}<0.05$, compared with the control group (PI3K); ${ }^{* *} \mathrm{P}<0.05$, compared with the control group (AKT); ${ }^{\# \#} \mathrm{P}<0.05$, compared with the control group $(\mathrm{CEBP} \alpha)$. PI3K, phosphoinositide-3 kinase; CEBP $\alpha$, CCAAT/enhancer binding protein $\alpha$.

for osteogenic differentiation increased, while the capacity for adipogenic differentiation decreased following treatment with Sirt1 activator $(0.4 \mu \mathrm{M})$. Sirt1 treatment was observed to exert the highest effect on TSC differentiation on day 10.

Sirt1 drives osteogenic differentiation of TSCs via upregulating $\beta$-catenin and Runx 2 and represses adipogenic differentiation of TSCs through downregulating CEBP $\alpha$ and PPAR $\gamma$ and the $P I 3 K / A K T$ pathway. The present study showed that increases in Sirt1 expression in TSCs enhanced $\beta$-catenin and Runx2 expression, while the expression of PI3K, AKT, CEBP $\alpha$ and PPAR $\gamma$ was decreased (Fig. 4). Furthermore, treatment with Sirt1 inhibitor reduced not only Sirt1 expression but also $\beta$-catenin and Runx 2 expression, while enhancing the 
expression of PI3K, AKT, CEBP $\alpha$ and PPAR $\gamma$. These results indicated that Sirt1 promotes osteogenic differentiation of TSCs through upregulating $\beta$-catenin and Runx 2 expression, while it inhibits the expression of CEBP $\alpha$ and PPAR $\gamma$ as well as the PI3K/AKT pathway, thereby inhibiting the adipogenic differentiation of TSCs $(\mathrm{P}<0.05)$.

\section{Discussion}

Sirtl is an NAD-dependent deacetylase which is important in numerous biological processes, including cell cycle regulation, cell differentiation, cell survival and apoptosis. Sirt1 regulates osteogenic differentiation of mesenchymal stem cells by deacetylating H3K9 in the promoter sequence of the Sost gene, which encodes sclerostin (10).

The present study confirmed that Sirt1 is expressed in TSCs and revealed that it enhances their osteogenic differentiation capacity in a time-dependent manner. Furthermore, the gene and protein levels of osteogenesis marker BMP2, were elevated by Sirt1 in a time-dependent manner. Peak values of BMP2 expression were observed on day 10 followed of treatment with Sirt1 activator. Conversely, the osteogenic differentiation capacity of TSCs was decreased after treatment with Sirt1 inhibitor. By contrast, treatment with Sirt1 activator reduced the adipogenic capacity of TSCs, while it was increased by the Sirt1 inhibitor.

Recently, Simic et al (16) showed that Sirtl deacetylates Runx 2 and $\beta$-catenin to regulate the differentiation of mesenchymal stem cells. Sirt1 activated by resveratrol was reported to promote osteogenic differentiation of mesenchymal stem cells by increasing Runx 2 expression, increasing its combination with PPAR $\gamma$ and inhibiting the activity of PPAR $\gamma$ through its cofactor nuclear receptor co-repressor 1 (NCoR1) (14). PPAR $\gamma$ has an important role in the balance of adipogenic and osteogenic differentiation of mesenchymal stem cells (15). Overexpressed Sirt1 can be anchored to NCoR1 and -2, the inhibitory co-factors of PPAR $\gamma$, which in turn suppresses the expression of PPAR $\gamma$ and $\mathrm{C} / \mathrm{EBP} \alpha$ and further inhibits the cytopoiesis of adipocytes (i.e. it reduces the transformation of pre-adipocytes into adipocytes) (14). Furthermore, Sirt1 can inhibit the transcriptional activity of AKT2 and interact with AKT2 and CEBP $\alpha$ (16). Silencing of AKT2 and/or Sirtl was shown to affect pre-adipogenesis through the PI3K/AKT pathway, which exhibits a synergy with the CEBP $\alpha$ pathway (16). The present study explored whether Sirtl regulates the differentiation of TSCs in a similar manner to that of mesenchymal stem cells.

In the present study, it was revealed that increased Sirt1 expression enhanced $\beta$-catenin and Runx 2 expression in TSCs and reduced the expression of PI3K, AKT, CEBP $\alpha$ and PPAR $\gamma$, while treatment with Sirt1 inhibitor had the opposite effect. It is therefore concluded that Sirt1 promotes osteogenic differentiation of TSCs through upregulation of $\beta$-catenin and Runx2, while inhibiting adipogenic differentiation of TSCs via inhibiting the PI3K/AKT pathway as well as the expression of CEBP $\alpha$ and PPAR $\gamma$.

In conclusion, to the best of our knowledge, the present study was the first to report that Sirt1 is endogenously expressed in TSCs. Sirtl was demonstrated to time-dependently promote the osteogenic differentiation of TSCs through upregulating $\beta$-catenin and Runx 2 and to inhibit the adipogenic differentiation of TSCs through inhibiting the PI3K/AKT pathway with downregulation of $\mathrm{CEBP} \alpha$ and PPAR $\gamma$. During the process of tendon injury repair, TSCs favor differentiation into cartilage and bone, while the accumulation of adipose tissue is not conducive to the recovery from tendinopathy. Therefore, by targeting Sirt1, it may be possible to regulate osteogenic differentiation of TSCs as well as adipose accumulation in injured tendons. Upregulation of Sirt1 may be an efficient strategy for the treatment of refractory tendinopathy using TSCs. This approach may represent a breakthrough leading to the development of novel clinical techniques with the potential to fundamentally improve the treatment of sports injuries.

\section{Acknowledgements}

The present study was supported by the National Natural Science Foundation of China (grant nos. 81230040 and 81401831).

\section{References}

1. Mahieu NN, Witvrouw E, Stevens V, Van Tiggelen D and Roget P: Intrinsic risk factors for the development of achilles tendon overuse injury: A prospective study. Am J Sports Med 34: 226-235, 2006.

2. Maffulli N: Achilles tendon pathology, present and future. $\mathrm{Br}$ J Sports Med 45: e2 doi:10.1136/bjsm.2010.081570.18, 2011.

3. Zhang $\mathrm{J}$ and Wang $\mathrm{JH}$ : Characterization of differential properties of rabbit tendon stem cells and tenocytes. BMC Musculoskelet Disord 11: 10, 2010.

4. Bi Y, Ehirchiou D, Kilts TM, Inkson CA, Embree MC, Sonoyama W, Li L, Leet AI, Seo BM, Zhang L, et al: Identification of tendon stem/progenitor cells and the role of the extracellular matrix in their niche. Nat Med 13: 1219-1227, 2007.

5. Rui YF, Lui PP, Li G, Fu SC, Lee YW and Chan KM: Isolation and characterization of multipotent rat tendon-derived stem cells. Tissue Eng Part A 16: 1549-1558, 2010.

6. Zhang J and Wang JH: Production of PGE(2) increases in tendons subjected to repetitive mechanical loading and induces differentiation of tendon stem cells into non-tenocytes. J Orthop Res 28: 198-203, 2010.

7. de Mos M, Koevoet WJ, Jahr H, Verstegen MM, Heijboer MP, Kops N, van Leeuwen JP, Weinans H, Verhaar JA and van Osch GJ: Intrinsic differentiation potential of adolescent human tendon tissue: An in-vitro cell differentiation study. BMC Musculoskelet Disord 8: 16, 2007.

8. Salingcarnboriboon R, Yoshitake H, Tsuji K, Obinata M, Amagasa T, Nifuji A and Noda M: Establishment of tendon-derived cell lines exhibiting pluripotent mesenchymal stem cell-like property. Exp Cell Res 287: 289-300, 2003.

9. Blander G and Guarente L: The Sir2 family of protein deacetylases. Annu Rev Biochem 73: 417-435, 2004.

10. Cohen-Kfir E, Artsi H, Levin A, Abramowitz E, Bajayo A, Gurt I, Zhong L, D'Urso A, Toiber D, Mostoslavsky R and Dresner-Pollak R: Sirt1 is a regulator of bone mass and a repressor of Sost encoding for sclerostin, a bone formation inhibitor. Endocrinology 152: 4514-4524, 2011.

11. Backesjo CM, Li Y, Lindgren U and Haldosén LA: Activation of Sirtl decreases adipocyte formation during osteoblast differentiation of mesenchymal stem cells. Cells Tissues Organs 189: 93-97, 2009.

12. Liu J, Chen L, Tao X and Tang K: Phosphoinositide 3-kinase/Akt signaling is essential for prostaglandin E2-induced osteogenic differentiation of rat tendon stem cells. Biochem Biophys Res Commun 435: 514-519, 2013

13. Liu J, Chen L, Zhou Y, Liu X and Tang K: Insulin-like growth factor-1 and bone morphogenetic protein-2 jointly mediate prostaglandin e2-induced adipogenic differentiation of rat tendon stem cells. PloS one 9: e85469, 2014.

14. Hata K, Nishimura R, Ikeda F, Yamashita K, Matsubara T, Nokubi T and Yoneda T: Differential roles of Smad1 and p38 kinase in regulation of peroxisome proliferator-activating receptor gamma during bone morphogenetic protein 2 -induced adipogenesis. Mol Biol Cell 14: 545-555, 2003. 
15. Ji C, Chang W, Centrella M and McCarthy TL: Activation domains of CCAAT enhancer binding protein delta: Regions required for native activity and prostaglandin E2-dependent transactivation of insulin-like growth factor I gene expression in rat osteoblasts. Mol Endocrinol 17: 1834-1843, 2003.
16. Simic P, Zainabadi K, Bell E, Sykes DB, Saez B, Lotinun S, Baron R, Scadden D, Schipani E and Guarente L: SIRT1 regulates differentiation of mesenchymal stem cells by deacetylating $\beta$-catenin. EMBO Mol Med 5: 430-440, 2013. 